American Journal of Applied Sciences 5 (12): 1670-1675, 2008

ISSN 1546-9239

(C) 2008 Science Publications

\title{
Power Enhancement of Weightlifters during Snatch through Reducing Torque on Joints by Particle Swarm Optimization
}

\author{
Firooz Salaami, Nima Jamshidi, Mostafa Rostami and Siamak Najarian \\ Faculty of Biomedical Engineering, Amirkabir University of Technology, Tehran, Iran
}

\begin{abstract}
In this research, an athlete's body on sagittal plane in tension phase of snatch weightlifting has been modeled in two dimensions for calculating the generated torques in joints. The error back propagation multi-layer perceptrons has been used for modeling the torque through changing the angular velocity, angular acceleration and absolute angle of each segment. Finally, the torque in joints has been minimized by particle swarm optimization technique and the power of athlete has been maximized. The method of weightlifting has been captured by high speed camera and the films have been analyzed through motion analysis software. Consequently, the required kinematic data for mathematical model of weightlifter has been produced. Unlike previous research reports, the technique of weightlifting has been modified with the aid of artificial neural network modeling to enhance athlete's power, instead of optimizing the effect of body parameters and sport facilities. In addition, this study focuses on computational intelligent techniques for optimization instead of classical methods.
\end{abstract}

Key words: Neural network, technique, sport, biomechanics

\section{INTRODUCTION}

Study of athletic movement from biomechanical view is one of the important research fields. Majority of research in this field is for recognition and optimization of movement. The recognition requires a true definition of a dynamic model of athlete's body with respect to biomechanical limitations. In order to find out the optimized athletic movement, various optimization methods have been applied. In this research, a new application of artificial intelligence in the scope of sport biomechanics has been introduced which has a wide application for optimization of athlete's movement in various sports. The findings of this research significantly improve the recognition and optimization of athlete's movement and prompt the rank of athlete's in global championships. Previous studies on the dynamic model of weightlifting are being briefly mentioned in the following part. A mathematical model on the sagittal plane was defined by five links. Through received information from camera and Newton-Euler formula, the values of force and torque were determined during snatch movement in the tension phase $^{[1]}$. The bar trajectory and joint angles of athletes with different anthropometric factors were analyzed ${ }^{[2]}$. A new procedure for calculating the power production during Olympic lifting movements was developed ${ }^{[3]}$. Data obtained from 16-mm film of weightlifters were analyzed to study energy changes during body segment and barbell movements, energy transfer to the barbell and energy transfer between segments during the lifting movements contested. The results provided a detailed understanding of the magnitude and temporal input of energy from dominant muscle groups during a lift ${ }^{[4]}$. A multi segment model of the lifters' movement in the sagittal plane was developed by using equations of motion, force and moments from applied data film. Analysis was limited to body segment orientations, vertical bar accelerations, vertical joint reaction forces, segmental angular accelerations, horizontal moment arms of the bar to selected joints and inter-segmental resultant moments. Maximum vertical bar acceleration and angular acceleration of the trunk tended to occur near lift-off in the skilled lifters. Within each subject, the hip joint experienced the greatest torque because of the relatively large horizontal moment arm of the bar to this joint ${ }^{[5]}$. Escamilla conducted a research to quantify biomechanical parameters employing two-dimensional and three-dimensional analyses while performing the squat with varying stance widths ${ }^{[6]}$. Ankle plantar flexor (10-51 N.m), knee extensor (359-573 N.m) and hip extensor (275-577 N.m) net muscle moments were

Corresponding Author Dr. Mostafa Rostami, Assistant Professor, No. 424, Hafez Avenue, Department of Biomechanics, Faculty of Biomedical Engineering, Amirkabir University of Technology, Tehran, P.O. Box 15875-4413, Iran Tel: (+98-21)-6454-2378 Fax: (+98-21)-6646-8186 
Am. J. Applied Sci., 5 (12): 1670-1675, 2008

generated for the narrow stance squat, whereas ankle dorsiflexor (34-284 N.m), knee extensor (447-756 N.m) and hip extensor (382-628 N.m) net muscle moments were generated for the medium stance squat and wide stance squat. Later, the snatch movements of two female athletes were examined in junior world weightlifting competition by means of camera techniques and through motion analysis software, speed versus time and bar trajectory were determined ${ }^{[7]}$.

In previous research studies, we minimized the generated torque during snatch movement by neural networks and fuzzy logic ${ }^{[8,9]}$. In majority of pervious works, the focus was on studying and modeling of movement in order to recognize and calculate kinematic parameters of weightlifter but there was less consideration given to power enhancement of weightlifter. It seems that the work on power enhancement can be divided in two branches: the first one focused on optimizing the effect of body parameters and sport facilities like weightlifters belt ${ }^{[10-14]}$ and the second focused on the technique modification of weightlifter through mathematical modeling and various optimization methods $^{[8,9,15-19]}$.

There are two approaches for optimization. The first approach is artificial intelligent. The other one is the optimal control and classical numerical method. Application of neural networks in the optimization of sport technique is a new discussion. The finding of this research will help the trainers to improve the technique of Olympic weightlifter.

\section{MATERIALS AND METHODS}

Mechanical modeling of body: Until now, various dynamic models with different inputs and outputs for simulation of human movement have been developed. Since internal forces and torques in joints could not be measured directly in a biological system, the kinematical and anthropometric parameters have been used because they were calculated indirectly. The mass of body segments have been determined through the Zatsirosky approach ${ }^{[20]}$. In order to estimate the length of each segment, Muftic et al. formulattions have been used $^{[21]}$. The mass calculation table of each segment have been computed based on Zatsirosky formulation in Matlab 7.0 in which the input variables are mass and height of the athlete. In order to estimate the moment of inertia around the center of mass, Chaffin et al. formula has been used ${ }^{[22]}$.

$$
\mathrm{I}=0.09 \mathrm{ML}^{2}
$$

Table 1: Estimating the mass, length, center of mass and moment of inertia of segments

\begin{tabular}{lllll}
\hline $\begin{array}{l}\text { Moment of } \\
\text { inertia }\left(\mathrm{kg} \mathrm{cm}^{-2}\right)\end{array}$ & $\begin{array}{l}\text { Center of } \\
\text { Mass }(\mathrm{m})\end{array}$ & $\begin{array}{l}\text { Length } \\
(\mathrm{m})\end{array}$ & $\begin{array}{l}\text { Mass } \\
(\mathrm{kg})\end{array}$ & $\begin{array}{l}\text { Segment } \\
\text { name }\end{array}$ \\
\hline 0.041875 & 0.2342 & 0.3934 & 3.3699 & $\begin{array}{l}\text { Shank } \\
0.272330\end{array}$ \\
0.2836 & 0.5202 & 11.182 & Thigh \\
0.589120 & 0.2965 & 0.6065 & 17.761 & Trunk \\
0.021777 & 0.1971 & 0.3318 & 2.1979 & Upper arm \\
0.066405 & 0.1074 & 0.2513 & 1.2796 & Lower arm \\
\hline
\end{tabular}

where, $\mathrm{M}$ is mass of segment and $\mathrm{L}$ is the length of the segment. The results of computation are shown in Table 1. The athlete of this research has $80(\mathrm{~kg})$ weight and $171(\mathrm{~cm})$ height. A $90(\mathrm{~kg})$ weight was lifted by the athlete.

Momentum calculation from dynamic model: In Fig. 1, the free force diagram of the athlete's body is shown. In this research, the athlete's body on sagittal plane with 5 segments has been considered. These 5 segments are ankle, knee, hip, shoulder and elbow. Foot joint has been considered as a reference segment. The body's mass, moment of inertia tensor and coordinates for the body's center of gravity and kinematic data are required for modeling and simulation of each segment.

These data have been used as inputs for our SimMechanic model. Through putting sensors on SimMechanic model and using the following equations, the desirable outputs including matrices of torque in joints have been calculated during a continuous modeling. It has been assumed that body is symmetric across the sagittal, the segments are considered as rigid links, the length of each segment is constant during the simulation, the friction in joints has been ignored and every joint has been considered as a revolute joint. The required kinematics data that were obtained from the film of weightlifters on sagittal plane were captured by a high speed camera that set at frame rate of $60 \mathrm{~Hz}$. Finally, the recorded data were analyzed by motion analysis software ${ }^{[23]}$.

In Fig. 2, the obtained kinematic data are presented. Dynamic Model has been solved by inverse kinematics method. A five link model on the sagittal plan, which has been designed for simulation of snatch movement by SimMechanic, has is shown in Fig. ${ }^{[24]}$. The next step is the modeling of the torque by artificial neural networks technique through changing the angular velocity, angular acceleration and absolute angle of each segment.

Artificial neural networks modeling: Neural networks have emerged as a very popular area of research, both from the design and the usage points of 


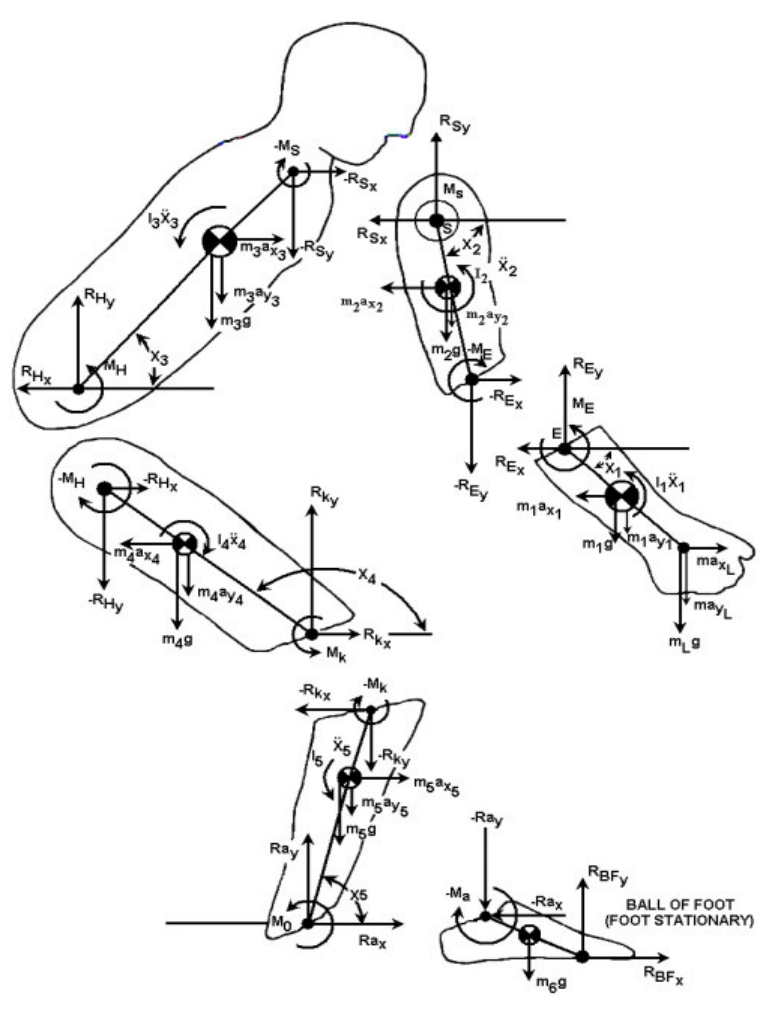

Fig. 1: Six-segment diagram of human's body ${ }^{[20]}$

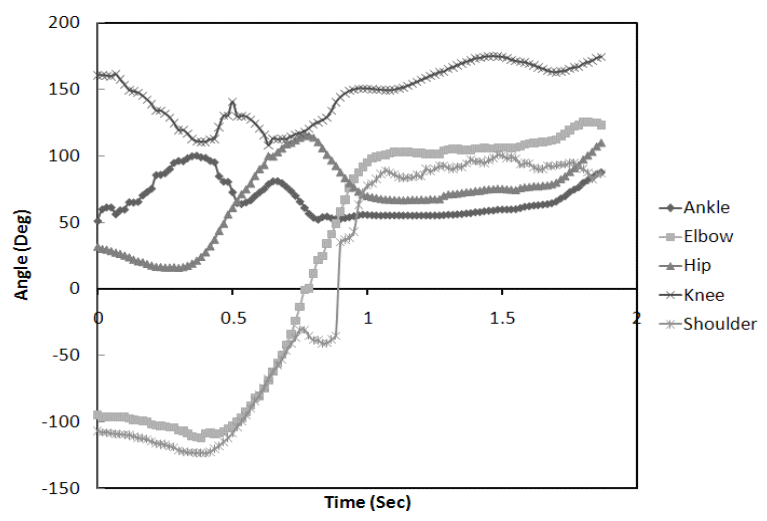

Fig. 2: Absolute angle variations of each joint

view $^{[26]}$. There is a great amount of academic interest in the applications of neural networks in engineering but there is a lack in sports fields. The network used in this research is the error back propagation multi-layer perceptrons network which has a high ability to solve nonlinear problems because of its nonlinear transfer function but the weakness of this algorithm is trapping of network in a local minimum. In order to prevent this, Haffman and Carpenter's formula has been used to estimate the number of neurons.

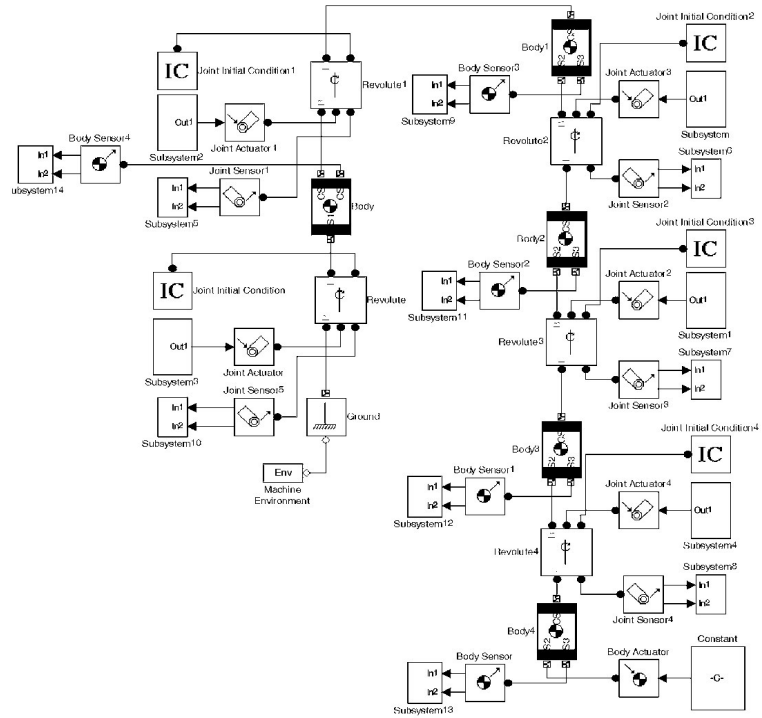

Fig. 3: A five-link model on the sagittal plan has been designed for an athlete by SimMechanics for Weightlifting (Snatch)

$$
\mathrm{n}_{\mathrm{s}}=\beta\left\{\mathrm{n}_{\mathrm{h}}\left(\mathrm{n}_{\mathrm{i}}+1\right)+\mathrm{n}_{\mathrm{o}}\left(\mathrm{n}_{\mathrm{h}}+1\right)\right\}
$$

where, $n_{s}$ is the number of the pairs which need to be trained, $n_{h}$ is the number of hidden layer neurons, $n_{i}$ is the number of input layer neurons and $n_{o}$ is the number of output layer neurons. The constant of $\beta$ is related to the degree of over-determination. This formula has been used for determining limit of the neurons in each layer $^{[25]}$.

Levenberg-marquardt algorithm ${ }^{[25]}$ : The LevenbergMarquardt algorithm was designed to approach secondorder training speed computing the Hessian matrix. When the performance function has the form of a sum of squares, then the Hessian matrix can be approximated as:

$$
\mathrm{H}=\mathrm{J}^{\mathrm{T}} \mathrm{J}
$$

and the gradient can be computed as:

$$
\mathrm{g}=\mathrm{J}^{\mathrm{T}} \mathrm{e}
$$

where, $\mathrm{j}$ is the Jacobian matrix that contains first derivatives of the network errors with respect to the weights and biases and $\mathrm{e}$ is a vector of network errors. The Jacobian matrix can be computed through a standard back propagation technique that is much less complex than computing the Hessian matrix. The 
Am. J. Applied Sci., 5 (12): 1670-1675, 2008

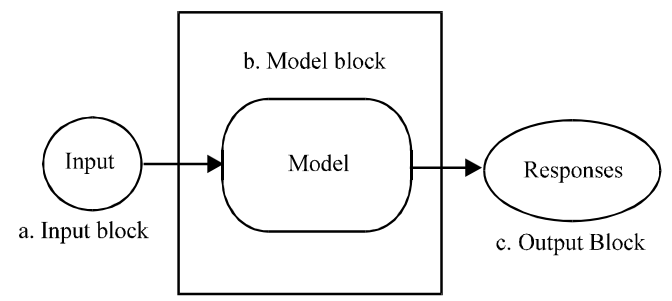

Fig. 4: Test plan in mbcmodel of GUI

Lavender Marquardt algorithm uses this approximation for the Hessian matrix in the following Newton-like update:

$$
\mathrm{X}_{\mathrm{k}+1}=\mathrm{X}_{\mathrm{k}}\left|\mathrm{J}^{\mathrm{T}} \mathrm{J}+\mu \mathrm{I}\right| \mathrm{J}^{\mathrm{T}} \mathrm{e}
$$

When the scalar $\mu$ is zero, this is just the Newton's method, using the approximate Hessian matrix. When $\mu$ is large, this becomes gradient descent with a small step size. The Newton's method is faster and more accurate near an error minimum, so the aim is to shift towards the Newton's method as quickly as possible. Thus $\mu$ is decreased after each successful step (reduction in performance function) and is increased only when a tentative step would increase the performance function. In this way, the performance function will always be reduced in each iteration of the algorithm.

Implementation neural network modeling: Modelbased Calibration software (mbcmodel) of Matlab 7.0.4 has been used to obtain the model of neural networks ${ }^{[25]}$. Test plan in mbcmodel of GUI for estimation is shown in Fig. 4. The input block is kinematics data, $\mathrm{b}$ is the model block (the determination of the type of model is performed in this research) and c is the output block. Our output is the created torque in the joints. The input data of this model are kinematics data which are related to ankle, knee, hip, shoulder and elbow. These data included absolute joint angles of each segment to the horizon, absolute angular velocity and absolute angular acceleration. Outputs are torque on joints.

There are two stages for creating the network. Stage one is for training the network and the second stage is for testing the accuracy of previous stage. First, a group of input vectors are divided in two parts. One part is for training the networks and the other one applies for testing the accuracy and training until the error of test has been minimized. This approach has two advantages: one is prevention of the network from overfit. Second is enabling the network for accurate estimation by adding new input and output data. The model has been used in neural network with LevenbergMarquardt algorithm and has two hidden layers. The number of neurons of each hidden layer is, respectively, 10 and 5. Each vector input has 113 elements and the output is a vector with the same quantity of elements including joint torque. The train data are 90 and the test data are assumed to be 23. The value of Epochs is 3000 . The stages of training are 12. The hierarchical Epochs for Levenberg-Marquardt algorithm are 1000. Finally, $\beta$ is about 1.2 .

Optimization: Particle Swarm Optimization (PSO) is a population based stochastic optimization technique developed by Dr. Eberhart and Dr. Kennedy in 1995, inspired by social behavior of bird flocking or fish schooling. PSO is initialized with a group of random particles (solutions) and then searches for optima by updating generations. In every iteration, each particle is updated by following two best values. The first one is the best solution (fitness) it has achieved so far. The fitness value is also stored. This value is called pbest. Another best value that is tracked by the particle swarm optimizer is the best value, obtained so far by any particle in the population. This best value is a global best and called gbest. When a particle takes part in the population as its topological neighbors, the best value is a local best and is called lbest. After finding the two best values, the particle updates its velocity and positions with following equations:

$$
\begin{gathered}
\mathrm{v}[]=\mathrm{v}[]+\mathrm{c} 1 * \operatorname{rand}() *(\operatorname{pbest}[]-\operatorname{present}[]) \\
+\mathrm{c} 2 * \operatorname{rand}() *(\operatorname{gbest}[]-\operatorname{present}[]) \\
\operatorname{present}[]=\text { persent }[]+\mathrm{v}[]
\end{gathered}
$$

$\mathrm{v}[]$ is the particle velocity, persent[] is the current particle (solution). pbest[] and gbest[] are defined as stated before. rand () is a random number between $(0,1) . \mathrm{c} 1, \mathrm{c} 2$ are learning factors. Usually $\mathrm{c} 1=\mathrm{c} 2=2^{[27]}$

The next step after modeling by artificial neural networks technique is optimization of torques by pso method through changing the angular velocity and angular acceleration of each segment. The training targets are the weighting factors which are extracted from the model.

The aim is changing the angular velocity, i.e., angular acceleration of each segment for minimizing the generated torques on joints. The value of Vmax and Epochs are 2.1 and 150, respectivly. Our criterion for estimation of goodness is gbest. Original error is criterion for starting the optimization algorithm and gbest for optimization algorithm started from this point. The numbers of optimization particles are between 24 to 30 . 


\section{RESULTS AND DISCUSSION}

As shown in Fig. 5 the torque has been declined through Particle swarm optimization method. In fact, the reduction of torques in all joints of athlete leads to lift a heavier weight. Figure 6 illustrates the experimental angular velocity of each joint. Also, Fig. 7 shows the optimized angular velocity. A comparison between Fig. 6 and 7 reveals that there are limited variations between optimal and experimental angular velocity.

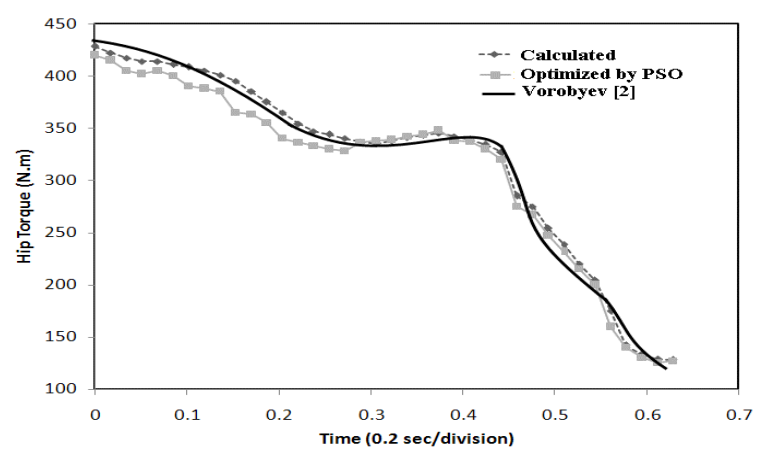

Fig. 5: Comparison between calculated torque by Vorobyev and calculated and optimized torque in our model

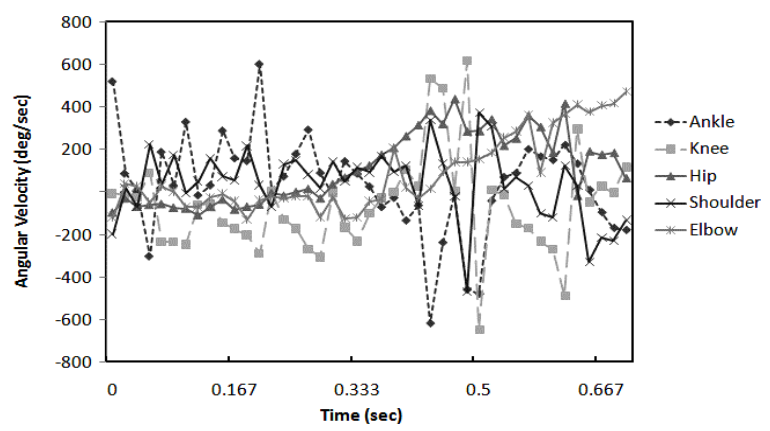

Fig. 6: Angular velocity until the start of catch

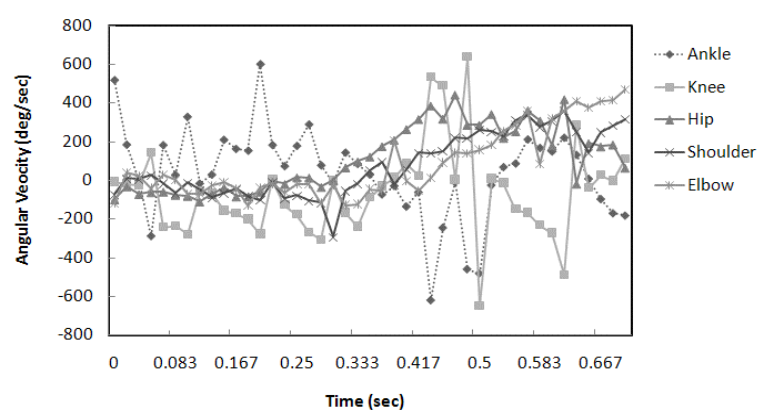

Fig. 7: Optimized angular velocity until the start of catch

\section{CONCLUSION}

The result of this research indicates that a little change in angular velocity in joints leads to considerable power enhancement of weightlifter. The finding of this research can help Olympic weightlifters to modify their techniques and enhance their power by defining the optimum angular velocity. It is recommended to extend the presented model by adding muscular models through combinations of springs and dampers which their coefficients can be obtained from electromyography.

\section{ACKNOWLEDGMENTS}

The authors gratefully acknowledge the Center of Excellence of Biomedical Engineering of Iran located at the Faculty of Biomedical Engineering of Amirkabir University of Technology, for their financial assistance in conducting this project.

\section{REFERENCES}

1. Garhammer, J., 1978. Biomechanical Analysis of Selected Snatch Lifts at the U.S. Senior National Weightlifting Champions. In Biomechanics of Sports and Kinanthropometry. Symposia Specialist Inc, Miami, Florida, pp: 475-484.

2. Vorobyev, A.N., 1978. A Textbook on Weightlifting. W.J. Brice Translation, International Weightlifting Federation, Budapest.

3. Garhammer, J., 1980. Power production by olympic weightlifters. Med. Sci. Sports Exercise, 12 (1): 54-60.

4. Garhammer, J., 1982. Energy flow during olympic weightlifting. Med. Sci. Sports Exercise, 14 (5): 353-360.

5. Brown, E.W. and K. Abani, 1985. Kinematics and kinetics of the dead lift in adolescent power lifters. Med. Sci. Sports Exercise, 17 (5): 554-66.

6. Escamilla, R.F., G.S. Fleisig, T.M. Lowry, S.W. Barrentine and J.R. Andrews, 2001. A threedimensional biomechanical analysis of the squat during varying stance widths. Med. Sci. Sports Exercise, 33 (6): 984-98.

7. Garhammer, J., 2001. Barbell trajectory velocity and power changes six attempts and four world record. Weightlifting USA, 19 (3): 27-30.

8. Jamshidi, N., M. Rostami, A. Arshi and F. Salaami, 2006. Weightlifting (Snatch) optimization by artificial neural networks. The 4th IASTED International Conference on Biomechanics, Spain. 
9. Jamshidi, N., M. Rostami and A. Arshi, 2007. Weightlifting (Snatch) modeling simulation and optimization by fuzzy logic. The 13th Iranian Conference on Biomedical Engineering, Sharif University of Technology, Tehran, Iran.

10. Fry, A.C., M.D. Ciroslan, M.D. Fry, C.D. LeRoux, B.K. Schilling and L.Z. Chiu, 2006. Anthropometric and performance variables discriminating elite american junior men weightlifters. J. Strength Conditioning Res., 20 (4): 861-866.

11. Markovic, G. and D. Sekulic, 2006. Modeling the influence of body size on weightlifting and powerlifting performance. Collegium Antropologicum, 30 (3): 607-613.

12. Kingma, I., G.S. Faber, E.K. Suwarganda, T.B. Bruijnen, R.J. Peters and J.H. Van Dieen, 2006. Effect of a stiff lifting belt on spine compression during lifting. Spine, 31 (22): E833-E839.

13. Cleather, D.J., 2006. Adjusting powerlifting performances for differences in body mass. J. Strength Conditioning Res., 20 (2): 412-421.

14. Stone, M.H., W.A. Sands, K.C. Pierce, J. Carlock, M. Cardinale and R.U. Newton, 2005. Relationship of maximum strength to weightlifting performance. Med. Sci. Sports Exercise, 37 (6): 1037-1043.

15. Nejadian, S.L. and M. Rostami, 2007. Optimization of barbell trajectory during the snatch lift technique by using genetic algorithm. BioMech 2007, Honolulu, Hawaii, USA.

16. Nejadian, S.L. and M. Rostami, 2007. Mathematical Modeling and Optimization of Snatch Lift Technique. 25th International Symposium on Biomechanics in Sports, Ouro Preto, Brazil.
17. Nejadian, S.L. and M. Rostami, 2007. Optimization of Barbell Trajectory during the Snatch Lift Technique by Using Genetic Algorithm. BioMech 2007, Honolulu, Hawaii, USA.

18. Rostami, M. and G. Bessonnet, 2001. Sagittal gait of a biped robot during the single support phase. Part 2: Optimal Motion. Robotica, 19: 241-253.

19. Nejadian, S.L., M. Rostami and F. Towhidkhah, 2008. Optimization of barbell trajectory during the snatch lift technique by using optimal control theory. Am. J. Applied Sci., 5 (5): 524-531.

20. Zatsirosky, M.V., 2002. Kinematics of Human Motion. Human Kinetics, United States of America.

21. Muftic, O. and M. Seif, 1998. Modeling of Biomechanical Systems. Hormozgan University Publication Center, Hormozgan University.

22. Chaffin DB and G.B.J. Andersson, 1991. Occupational Biomechanics. John Wily \& Sons, New York.

23. WINanalyze, Mikromak Service Brinkmann, Retrieved June 5, 2006. http://www.winanalyze. com/index.htm.

24. SimMechanics, The MathWorks Inc., Retrieved June 8, 2007. http://www.mathworks.com/access/ helpdesk/help/toolbox/physmod/mech.

25. The MathWorks Inc., Retrieved June 8, 2007, from http://www.mathworks.com/access/helpdesk/help/t oolbox.

26. Ramesh, S. and R. Rampal. Neural Networks and Management Science, Retrieved January 1, 2008. http://itorms.iris.okstate.edu/nnpaper.html.

27. HU, X., 2006. PSO Tutorial, Retrieved January 8, 2008. http://www.swarmintelligence.org/ tutorials.php. 